\title{
First principles determination of some static and dynamic properties of the liquid $3 d$ transition metals near melting
}

\author{
B.G. del Rio, C. Pascual, O. Rodriguez, L.E. González, D.J. González \\ Departamento de Física Teórica, Universidad de Valladolid, 47011 Valladolid, Spain
}

Received January 31, 2020, in final form March 26, 2020

\begin{abstract}
We report an ab initio molecular dynamics simulation study of several static and dynamic properties of the liquid $3 d$ transition metals. The calculated static structure factors show qualitative agreement with the available experimental data, and its second peak displays an asymmetric shape which suggests a significant local icosahedral short-range order. The dynamical structure reveals propagating density fluctuations whose dispersion relation has been evaluated; moreover, its long wavelength limit is compatible with their respective experimental sound velocity. Results are reported for the longitudinal and transverse current spectral functions as well as for the respective dispersion relations. We also analyze the possible appearance of transverse-like low-energy excitations in the calculated dynamic structure factors. Several transport coefficients have been evaluated and compared with the available experimental data.
\end{abstract}

Key words: liquid metals, first principles calculations

\section{Introduction}

This paper presents recent $a b$ initio molecular dynamics simulation studies for a range of bulk static, dynamic and electronic properties of the liquid $3 d$ transition metals at thermodynamic conditions near melting. We review some of our previous calculations [1-5] along with several new results.

Despite the practical relevance of the $3 d$ transition metals, it is remarkable that the experimental information concerning their static and dynamic properties in the liquid state is rather scarce.

The static structure factor, $S(q)$, encompasses the information about the average short-range order of a liquid system, and was first obtained experimentally by Waseda and coworkers [6] in the decade of the 1970s for the $3 d$ transition metals, by means of X-ray diffraction (XD) techniques. More recently, the $S(q)$ of 1-Ti, l-Fe, 1-Ni and 1-Zn have been determined again by either XD or neutron diffraction (ND) experiments [7-11]. In 1-Zn, the new measurements showed a good agreement with the older ones, but for 1-Ni and l-Fe, the comparison with Waseda and coworkers' XD (WXD) data for the $S(q)$, revealed some discrepancies concerning the height of the main peak as well as the shape of the second peak; nevertheless, the positions of the maxima and minima of $S(q)$ were coincident in all the experiments. On the other hand, the recent XD and ND data for the $S(q)$ of 1-Ti [9, 10] yielded results well compatible between each other, but when compared with WXD data, they were displaced towards greater $q$-values by a significant amount of $\approx 0.20 \AA^{-1}$. Moreover, the recent XD data showed a second peak with a marked shoulder on the high- $q$ side; actually, shoulders on the high- $q$ side of the second peak of $S(q)$ have also been found experimentally in other transition metals $(\mathrm{Fe}, \mathrm{Ni}, \mathrm{Zr})[7,8,12]$. We think that these disparities call for new precise structural data for the other $3 d$ transition metals, both extracted from new additional experiments and/or from accurate simulations as the ab initio ones presented here.

Several transport coefficients of most of the $3 d$ liquid metals were reported in the literature [13--25]. The dynamic structure factors of 1-Ti, 1-Fe, 1-Ni and 1-Zn near melting were measured by both inelastic neutron (INS) and inelastic X-ray (IXS) scattering [17, 26-30], whereas the self dynamic structure factors 
of 1-Ni, 1-Ti and 1-Cu were determined by quasi-elastic neutron scattering [31--33]. There is, however, no experimental information about the dynamic structure of the rest of $3 d$ liquid metals.

To our knowlege, only l-Cu, l-Ni and l-Fe had been previously studied by other groups using ab initio simulation methods. For $1-\mathrm{Cu}$, three $a b$ initio studies evaluated its static properties at melting and some undercooled states [34, 36, 37]. As for 1-Ni, Jakse et al. [38, 39] studied a range of static and dynamic properties near melting and calculated the self-diffusion and shear viscosity coefficients. In the case of 1-Fe, Ganesh and Widom [40] analyzed several static properties, getting also into the undercooled region.

In our recent studies of the liquid $3 d$ transition metals [1-5], we were interested in the calculation and analysis of their dynamic properties. Besides its intrinsic interest, we were also motivated by the recent finding of some remarkable features such as low-energy transverse-like excitations in the dynamic structure factor and/or the appearance of a high-frequency branch in the transverse current dispersion relation, in addition to the usual one that appears at low frequencies. Indeed, the study of the microscopic processes behind the dynamic properties of liquid systems has been a long standing research field of Prof. Mryglod, and in this respect we spotlight his contributions to the development and application of the generalized collective modes (GCM) approach. As for the above mentioned features, there is still no clear explanation of their physical origin, although we have recently advanced some connection between the existence of a second branch in the transverse dispersion relation and the coupling of the transverse current with density fluctuations at all wavevectors [4, 41].

Our studies of the bulk liquid $3 d$ transition metals were performed with an $a b$ initio molecular dynamics (AIMD) simulation method based on the density functional theory [42, 43]. The liquid metal is modelled as an interacting system of ions and electrons where the ionic positions evolve according to classical mechanics while the electronic subsystem follows adiabatically. Table 1 provides information concerning the thermodynamic states as well as some technical details. The AIMD simulations were performed with the Quantum-ESPRESSO package [44, 45], excepting 1-Fe and 1-Zn for which we used the VASP code [46-49]. The generalized gradient approximation of Perdew-Burke-Ernzerhof [50] was used to account for the electronic exchange-correlation energy, with the exceptions of 1-Ti and 1-Cu for which we used Perdew and Wang's approximation [51] and Perdew and Zunger's local density expression [52], respectively.

The ion-electron interaction was described by ultrasoft pseudopotentials [53], excepting 1-Fe and 1-Zn for which the projector augmented wave all-electron description [54, 55] was used. For all the systems, the cutoff for the plane-wave representation of the orbitals was within the range 25.0-35.0 Ryd and the sampling of the Brillouin zone was performed by means of the single $\Gamma$ point.

We recall that most previous studies of the liquid $3 d$ transition metals were carried out by using semiempirical or more fundamental pair (or many-body) potentials coupled with liquid state theories or classical molecular dynamics simulations [56-59]. Moreover, in these approaches the electronic degrees

Table 1. Input data of the liquid $3 d$ transition metals considered in the present AIMD simulation study. $\rho$ is the total ionic number density, (taken from [57]), $T$ is the temperature, $N_{\text {part }}$ is the number of particles, $\Delta t$ is the ionic timestep, $Z_{\mathrm{val}}$ is the number of valence electrons and $N_{\mathrm{c}}$ is the total number of configurations.

\begin{tabular}{ccccccc}
\hline & $\rho\left(\AA^{-3}\right)$ & $T(\mathrm{~K})$ & $N_{\text {part }}$ & $\Delta t(\mathrm{ps})$ & $Z_{\mathrm{val}}$ & $N_{\mathrm{c}}$ \\
\hline $\mathrm{Sc}$ & 0.0391 & 1875 & 120 & 0.0045 & 11 & 29000 \\
$\mathrm{Ti}$ & 0.0522 & 2000 & 100 & 0.0045 & 12 & 30700 \\
$\mathrm{~V}$ & 0.0634 & 2173 & 120 & 0.0040 & 13 & 20000 \\
$\mathrm{Cr}$ & 0.0713 & 2173 & 100 & 0.0045 & 14 & 27000 \\
$\mathrm{Mn}$ & 0.0655 & 1550 & 120 & 0.0045 & 15 & 13200 \\
$\mathrm{Fe}$ & 0.0746 & 1873 & 100 & 0.0020 & 8 & 22100 \\
$\mathrm{Co}$ & 0.0787 & 1850 & 135 & 0.0045 & 9 & 13100 \\
$\mathrm{Ni}$ & 0.0792 & 1773 & 120 & 00055 & 10 & 21000 \\
$\mathrm{Cu}$ & 0.0755 & 1423 & 150 & 00050 & 11 & 25500 \\
$\mathrm{Zn}$ & 0.0616 & 723 & 90 & 00040 & 12 & 36000 \\
\hline
\end{tabular}


of freedom are hidden into the effective potential and, therefore, no electronic properties (i.e., density of states, conductivity, ... ) can be consistently/reliably calculated. On the contrary, the AIMD simulation method yields parameter-free interatomic interactions derived from first principles, and the valence electrons are explicitly taken into account during the calculation.

\section{Results and discussion}

The evaluation of static and dynamic properties of the bulk liquid metal was performed by using the total number of equilibrium configurations listed in table 1

\subsection{Static properties}

The calculated static structure factors, $S(q)$, are plotted in figure 1 along with the corresponding WXD data [6]. The figure also includes the more recent XD and ND data [7-11] for 1-Ti, 1-Fe, 1-Ni and 1-Zn.

Aside from 1-Sc and 1-Ti, the calculated $S(q)$ shows a good qualitative agreement with the WXD data. There are some slight disparities, namely: (i) a very small phase shift in 1-V, 1-Mn and 1-Co, (ii) the calculations predict an asymmetric shape for the second peak with a more or less marked shoulder, whereas the XD data display, for all systems, a symmetric second peak. Notice that shoulders on the high- $q$ side of the second peak of $S(q)$ have also been experimentally observed in 1-Ti, 1-Fe, 1-Ni and $1-\mathrm{Zr}$ [7-10, 12]; moreover, they become more marked upon undercooling [60,62].

As for 1-Ti, comparison with the recent XD and ND data [9, 10] for the $S(q)$, reveals an excellent agreement with the present resuts, including the shape of the second peak as well as the shoulder on its high- $q$ side. Consequently, we believe that the WXD data for 1-Ti are somewhat unreliable and that something similar might also happen with 1-Sc, where an analogous (although opposite) dephasage is visible when compared with the present AIMD result.

From the calculated $S(q)$, within the range $q \leqslant 1.2 \AA^{-1}$, we used a least squares fit, $S(q)=s_{0}+s_{2} q^{2}$, to obtain an estimate for $S(q \rightarrow 0)$ and the results are given in table 2 . Then, we evaluated the isothermal compressibility, $\kappa_{T}$, by resorting to the relation $S(q \rightarrow 0)=\rho k_{\mathrm{B}} T \kappa_{T}$, where $k_{\mathrm{B}}$ is Boltzmann's constant. Table 2 lists the obtained results along with the available experimental data.

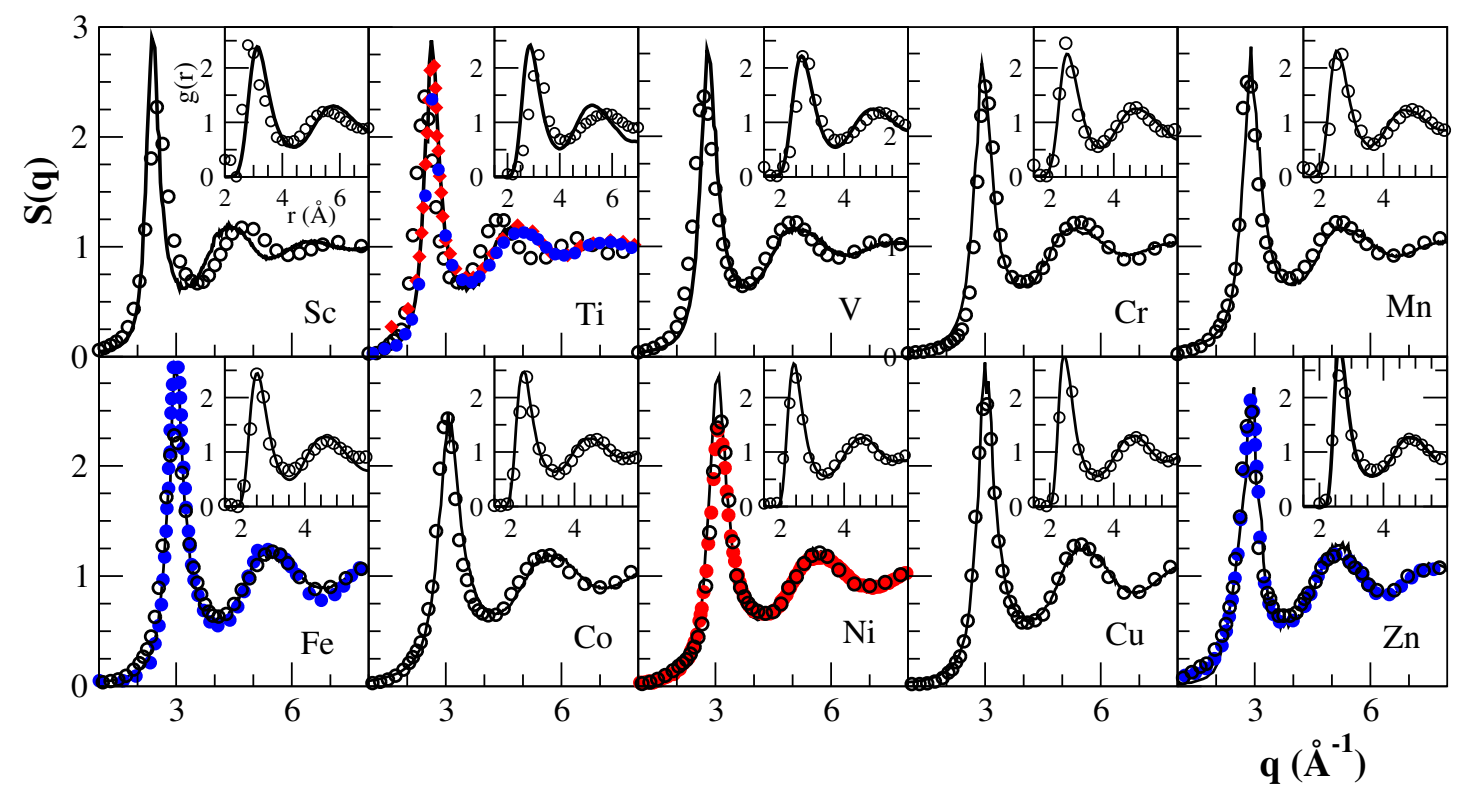

Figure 1. (Colour online) Static structure factor, $S(q)$, of the liquid $3 d$ transition metals. Continuous line: present AIMD calculations. Open circles: WXD data from [6]. The blue circles are XD data [7]-11] and red ones are ND data [7. 10]. The inset shows the corresponding pair distribution function. 
Table 2. Calculated values for $r_{\text {min }}$ (in $\AA$ ), coordination numbers $\mathrm{CN}, S(q \rightarrow 0)$ and isothermal compressibilities $\kappa_{T}$ (in $10^{-11} \mathrm{~m}^{2} \mathrm{~N}^{-1}$ units) for the liquid transition metals at the thermodynamic states given in table 1 The numbers in parenthesis are experimental and/or semiempirical data.

\begin{tabular}{cccccc}
\hline & $r_{\min }$ & $\mathrm{CN}$ & $S(q \rightarrow 0)$ & \multicolumn{2}{c}{$\kappa_{T}$} \\
\hline $\mathrm{Sc}$ & 4.27 & 12.8 & $0.020 \pm 0.002$ & $2.00 \pm 0.10$ & $\left(3.25^{a}\right)$ \\
$\mathrm{Ti}$ & 3.90 & 13.3 & $0.018 \pm 0.001$ & $1.28 \pm 0.10$ & $\left(1.67 \pm 0.02^{b}\right)$ \\
$\mathrm{V}$ & 3.62 & 12.6 & $0.021 \pm 0.002$ & $1.10 \pm 0.10$ & $\left(1.43^{a}, 1.21^{b}\right)$ \\
$\mathrm{Cr}$ & 3.47 & 12.5 & $0.020 \pm 0.002$ & $0.94 \pm 0.10$ & $\left(1.00^{a}, 1.10^{b}\right)$ \\
$\mathrm{Mn}$ & 3.48 & 12.3 & $0.069 \pm 0.003$ & $4.9 \pm 0.30 \quad\left(3.00^{a}, 3.74 \pm 0.02\right)$ \\
$\mathrm{Fe}$ & 3.42 & 12.5 & $0.024 \pm 0.002$ & $1.24 \pm 0.10 \quad(1.21 \pm 0.02)$ \\
$\mathrm{Co}$ & 3.25 & 11.6 & $0.022 \pm 0.002$ & $1.10 \pm 0.10 \quad\left(1.43^{a}, 1.18 \pm 0.02^{b}\right)$ \\
$\mathrm{Ni}$ & 3.27 & 11.8 & $0.018 \pm 0.002$ & $0.95 \pm 0.10 \quad\left(1.45^{a}, 1.04 \pm 0.02^{b}\right)$ \\
$\mathrm{Cu}$ & 3.28 & 11.8 & $0.016 \pm 0.002$ & $1.10 \pm 0.10 \quad\left(1.49^{b}\right)$ \\
$\mathrm{Zn}$ & 3.69 & 12.4 & $0.009 \pm 0.004$ & $1.52 \pm 0.60$ & $\left(2.34^{b}\right)$ \\
\hline$a[25]^{b}[13]$ & & & \multicolumn{2}{c}{}
\end{tabular}

In figure 1, we also depicted the associated pair distribution functions, $g(r)$, which are compared with their respective $\mathrm{XD}$ data [6]. The associated coordination number $(\mathrm{CN})$ was evaluated by integrating the radial distribution function, $4 \pi \rho r^{2} g(r)$, up to the position of its first minimum, $r_{\text {min }}$. Table 2 shows the obtained values for the CN's which are typical of simple liquid metals near their respective triple points [35].

A more detailed three-dimensional description of the short range order in these liquid metals is achieved by 0.8 applying the common neighbour analysis [63] (CNA) method. This method allows one to characterize the local environment surrounding each atomic pair that contributes to the peaks of the $g(r)$, in terms of the number 0.6 and properties of the common nearest neighbours of the pair under consideration. Each bonded pair of atoms is characterized by four indices, with the first index being 0.4 1 if the pair belongs to the first peak of $g(r)$, the second index stands for the number of common first neighbours, the third index is the number of bonds that connect those shared first neighbours and the fourth index discriminates 0.2 among those configurations having the same first three indices but a different topology. The CNA method allows one to discern different local structures such as FCC, HCP, BCC and ICOS. For example, the close-packed (CP) structures, FCC and HCP, are composed of 142x-type pairs, the BCC is typified by $144 \mathrm{x}$ and $166 \mathrm{x}$ pairs whereas the perfect ICOS is characterized by $155 \mathrm{x}$ pairs and the distorted ICOS is described by the $154 \mathrm{x}$ and $143 \mathrm{x}$ pairs.

The CNA study is performed on inherent structures in which the ions are brought to local minima of the potential energy surface. For each metal, this calculation was made for four/five ionic configurations, well separated in time $(\approx 15.0-20.0 \mathrm{ps})$, and the results were averaged. These are plotted in figure 2 where it is noticed that the five-fold symmetry dominates, as the sum of perfect and distorted ICOS structures varies between $\approx 59 \%(1-\mathrm{Sc})$ and $\approx 72 \%(1-\mathrm{Cr}, 1-\mathrm{Co})$ of the pairs. The amount of local BCC-type pairs is also remarkable as it ranges from $\approx 32 \%(1-\mathrm{Ti})$ to $\approx 6 \%(1-\mathrm{Zn})$ of the pairs. Finally, the CP-type pairs are almost absent in the early transition metals but become significant for Mn and for the late elements (Co to $\mathrm{Zn}$ ). This behaviour of the $\mathrm{CP}$ vs BCC local structures correlates with the corresponding phase diagrams of the elements, since Sc to Fe melt from a BCC phase, while Co to $\mathrm{Zn}$ do so from $\mathrm{CP}$ structures, either $\mathrm{FCC}(\mathrm{Co}, \mathrm{Ni}, \mathrm{Cu})$ or $\mathrm{HCP}(\mathrm{Zn})$. The phase diagram of $\mathrm{Mn}$ is rather complex, and the high temperature 
BCC phase only exists down to $100 \mathrm{~K}$ below the melting point, where it transforms into FCC, and this latter phase appears to somehow partially survive locally also after melting.

\subsection{Dynamic properties}

We evaluated several dynamic magnitudes and their associated time correlation functions. Due to the macroscopically isotropic behaviour of the fluid, those correlation functions with a $\vec{q}$-dependence are transformed into $|\vec{q}|$-dependent magnitudes.

\subsubsection{Single-particle dynamics}

We report results for the self-diffusion coefficient, $D$, which, along with the shear viscosity, plays an important role in the study of crystal nucleation and growth in metallic melts. We evaluated the velocity autocorrelation function, $Z(t)$, and the mean square displacement, $\delta R^{2}(t)$, of a tagged ion in the fluid, for all the $3 d$ liquid metals. The diffusion coefficient can be determined through both of these functions, and both approaches yielded practically the same result for $D$, which is given in table 3 along with the available experimental/semiempirical data.

Table 3. Calculated values of the self-diffusion coefficient, $D\left(\AA^{2} / \mathrm{ps}\right)$, adiabatic sound velocity $c_{\mathrm{S}}(\mathrm{m} / \mathrm{s})$ and shear viscosity $\eta(\mathrm{GPa} \cdot \mathrm{ps})$ for the liquid transition metals at the thermodynamic states given in table 1 The numbers in parenthesis are experimental and/or semiempirical data.

\begin{tabular}{cccc}
\hline & $D$ & $c_{\mathrm{s}}$ & $\eta$ \\
\hline $\mathrm{Sc}$ & $0.55 \pm 0.01$ & $4400 \pm 200$ & $2.10 \pm 0.10$ \\
$\mathrm{Ti}$ & $0.49 \pm 0.01\left(0.53 \pm 0.02^{a}\right)$ & $4640 \pm 100\left(4407 \pm 108^{b}\right)$ & $2.85 \pm 0.15\left(2.20^{c}\right)$ \\
$\mathrm{V}$ & $0.51 \pm 0.01$ & $4725 \pm 200\left(4742^{b}\right)$ & $3.30 \pm 0.20$ \\
$\mathrm{Cr}$ & $0.47 \pm 0.01$ & $4500 \pm 200\left(4298^{b}\right)$ & $3.70 \pm 0.20$ \\
$\mathrm{Mn}$ & $0.46 \pm 0.02$ & $3100 \pm 150\left(3381^{d}\right)$ & $2.50 \pm 0.20$ \\
$\mathrm{Fe}$ & $0.37 \pm 0.02\left(0.355^{e}\right)$ & $3950 \pm 150\left(3820 \pm 150^{f}\right)$ & $5.00 \pm 0.30\left(5.30^{g}\right)$ \\
$\mathrm{Co}$ & $0.60 \pm 0.01$ & $3700 \pm 150\left(4048^{d}\right)$ & $3.20 \pm 0.20\left(4.12^{h}, 3.60^{i}\right)$ \\
$\mathrm{Ni}$ & $0.38 \pm 0.01\left(0.380 \pm 0.006^{j}\right)$ & $4250 \pm 150\left(4040 \pm 150^{g}\right)$ & $4.10 \pm 0.20\left(4.37^{h}\right)$ \\
$\mathrm{Cu}$ & $0.27 \pm 0.01\left(0.37 \pm 0.006^{l}\right)$ & $3550 \pm 150\left(3481^{d}\right)$ & $4.80 \pm 0.20\left(4.38^{m}\right)$ \\
$\mathrm{Zn}$ & $0.23 \pm 0.01\left(0.24 \pm 0.006^{n}\right)$ & $3150 \pm 250\left(2849^{d}\right)$ & $2.40 \pm 0.30\left(3.50^{e}, p\right)$ \\
\hline${ }^{a}[17],{ }^{b}[14],{ }^{c}[18],{ }^{d}[13],{ }^{e}[19],{ }^{f}[20],{ }^{g}[21],{ }^{h}[15],{ }^{i}[16],{ }^{j}[32],{ }^{l}[33],{ }^{m}[22],{ }^{n}[23],{ }^{p}[24]$
\end{tabular}

\subsubsection{Collective dynamics}

In a liquid, the dynamics of density fluctuations is usually described by the intermediate scattering function, $F(q, t)$, which is defined as autocorrelation function of the microscopic wavevector-dependent density [35]. The Fourier transform (FT) of $F(q, t)$ into the frequency domain leads to the dynamic structure factor, $S(q, \omega)$, which can be directly measured by either INS and/or IXS experiments.

Another important collective magnitude is the current due to the overall motion of the particles. It is a vectorial magnitude which is usually split into its longitudinal and transverse components with respect to $\vec{q}$. Then, the longitudinal, $J_{\mathrm{L}}(q, t)$, and transverse $J_{\mathrm{T}}(q, t)$, current correlation functions are obtained as autocorrelation functions of the corresponding quantities [35]. Their time FT yields the associated spectra, $J_{\mathrm{L}}(q, \omega)$ and $J_{\mathrm{T}}(q, \omega)$, respectively.

In figures 34. we plotted, for some metals and for some range of $q$-values, the obtained AIMD results for the $F(q, t)$. For small $q$-values, the $F(q, t)$ show an oscillatory behaviour, indicative of wave propagation, which is superposed on another decaying component, indicative of relaxation modes. The oscillations become weaker with increasing $q$-values and disappear at around $q \approx 4 / 5 q_{p}$, where $q_{p}$ denotes the position of the main peak of $S(q)$. For larger $q$ values, the decaying component becomes dominant leading at $q \approx q_{p}$ to a very slow monotonous decay of the $F(q, t)$. The relative strength of both 

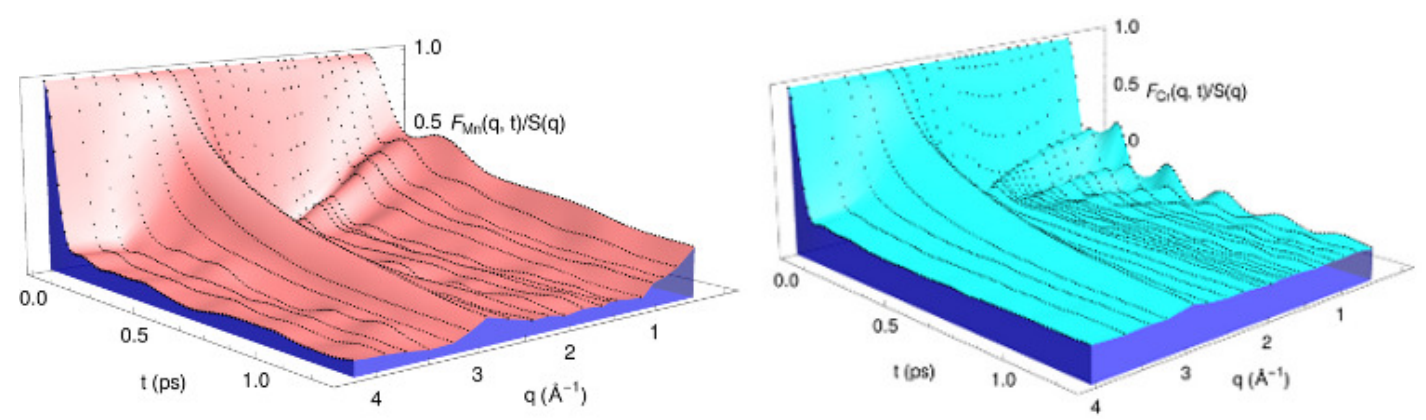

Figure 3. (Colour online) Intermediate scattering function, $F(q, t) / S(q)$, of 1-Mn at $T=1550 \mathrm{~K}$ and 1-Cr at $T=2173 \mathrm{~K}$ for several $q$ values.
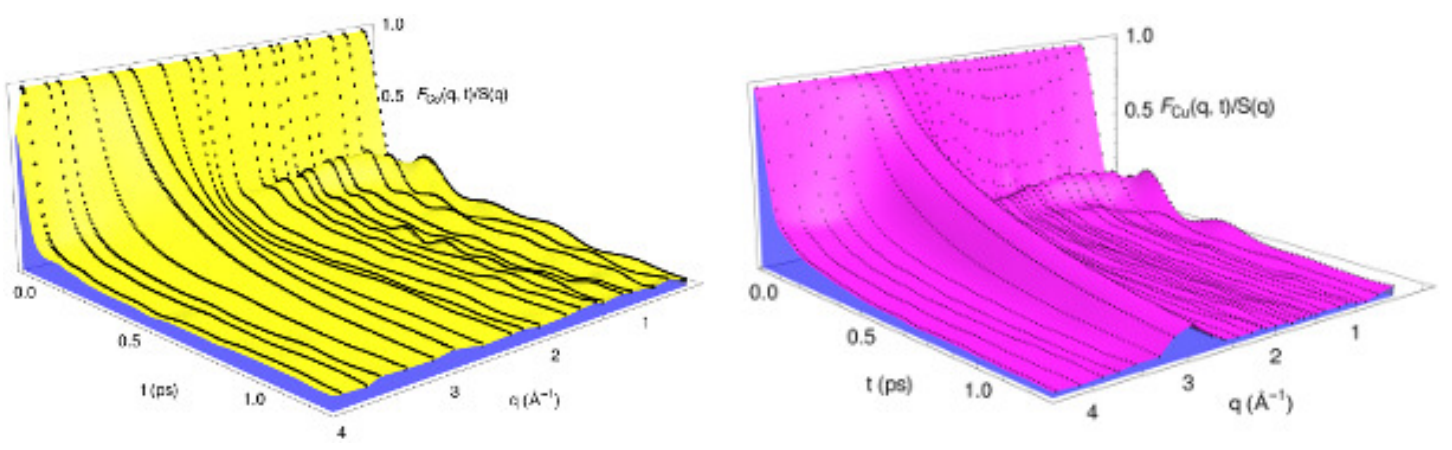

Figure 4. (Colour online) Same as the previous figure but for l-Co at $T=1850 \mathrm{~K}$ and $\mathrm{l}-\mathrm{Cu}$ at $T=1423 \mathrm{~K}$.

components depends on the system and we observe that 1-Mn has the weaker oscillations superposed on a very strong diffusive component.

Figures 5,6 show the associated $S(q, \omega)$. The behaviour of the calculated $S(q, \omega)$ is qualitatively similar for all the $3 d$ metals studied in this paper. Specifically, the $S(q, \omega)$ show side-peaks up to $q$-values $\approx(3 / 5) q_{p}$, which thereafter evolve into shoulders lasting up to $q \approx(4 / 5) q_{p}$. Therefrom, the $S(q, \omega)$ show a monotonous decreasing behaviour. Notice that for small $q$-values, the $S(q, \omega)$ of 1-Mn takes relatively large values when $\omega \rightarrow 0$; this is due to the important diffusive component of its $F(q, t)$ at those small $q$-values. From the positions of the side-peaks, $\omega_{m}(q)$, in the $S(q, \omega)$, a dispersion relation for the density excitations was obtained and is plotted in figure 7. In the hydrodynamic region (small $q$ ), the slope of the dispersion relation curve is the $q$-dependent adiabatic sound velocity $c_{\mathrm{s}}(q)=v_{\text {th }} \sqrt{\gamma / S(q)}$, with $v_{\text {th }}=\sqrt{k_{\mathrm{B}} T / m}$ being the thermal velocity and $\gamma$ being the ratio of specific heats. In the $q \rightarrow 0$ limit, the $c_{\mathrm{s}}(q)$ reduces to the bulk adiabatic sound velocity $c_{\mathrm{s}}$. However, the small size of the simulation box
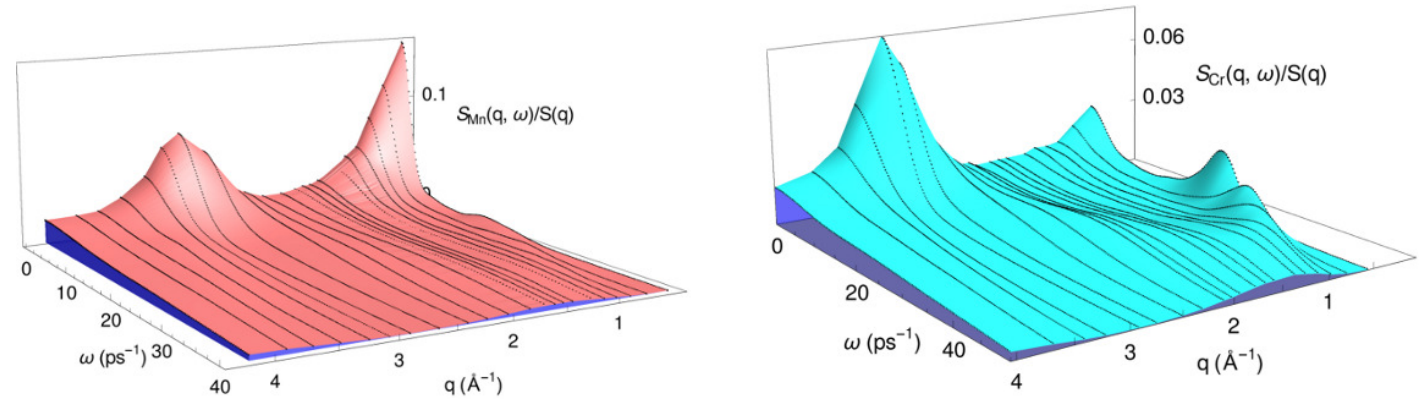

Figure 5. (Colour online) Dynamic structure factor, $S(q, \omega) / S(q)$, of 1-Mn at $T=1550 \mathrm{~K}$ and 1-Cr at $T=2173 \mathrm{~K}$ for several $q$ values. 

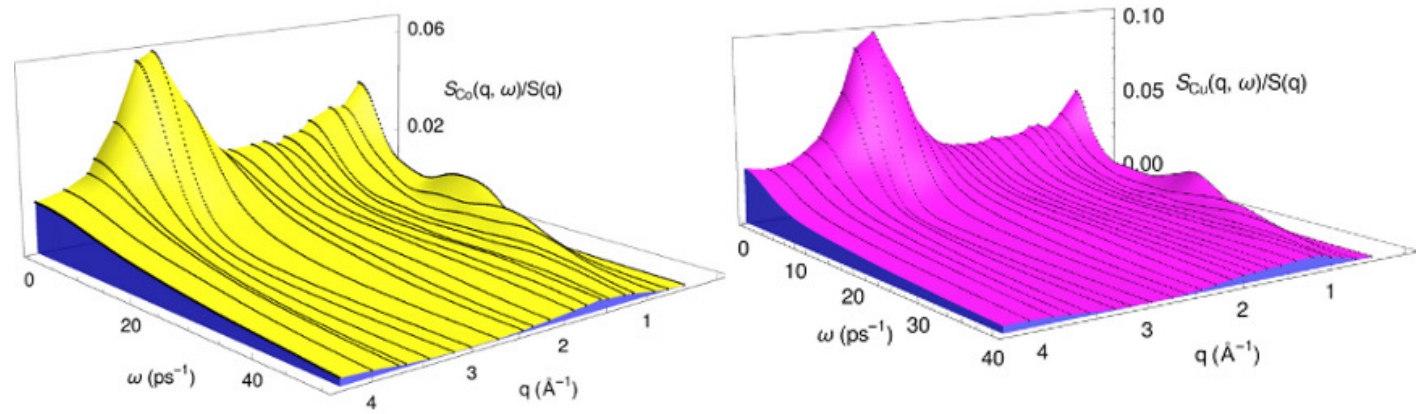

Figure 6. (Colour online) Same as the previous figure but for l-Co at $T=1850 \mathrm{~K}$ and l-Cu at $T=1423 \mathrm{~K}$.

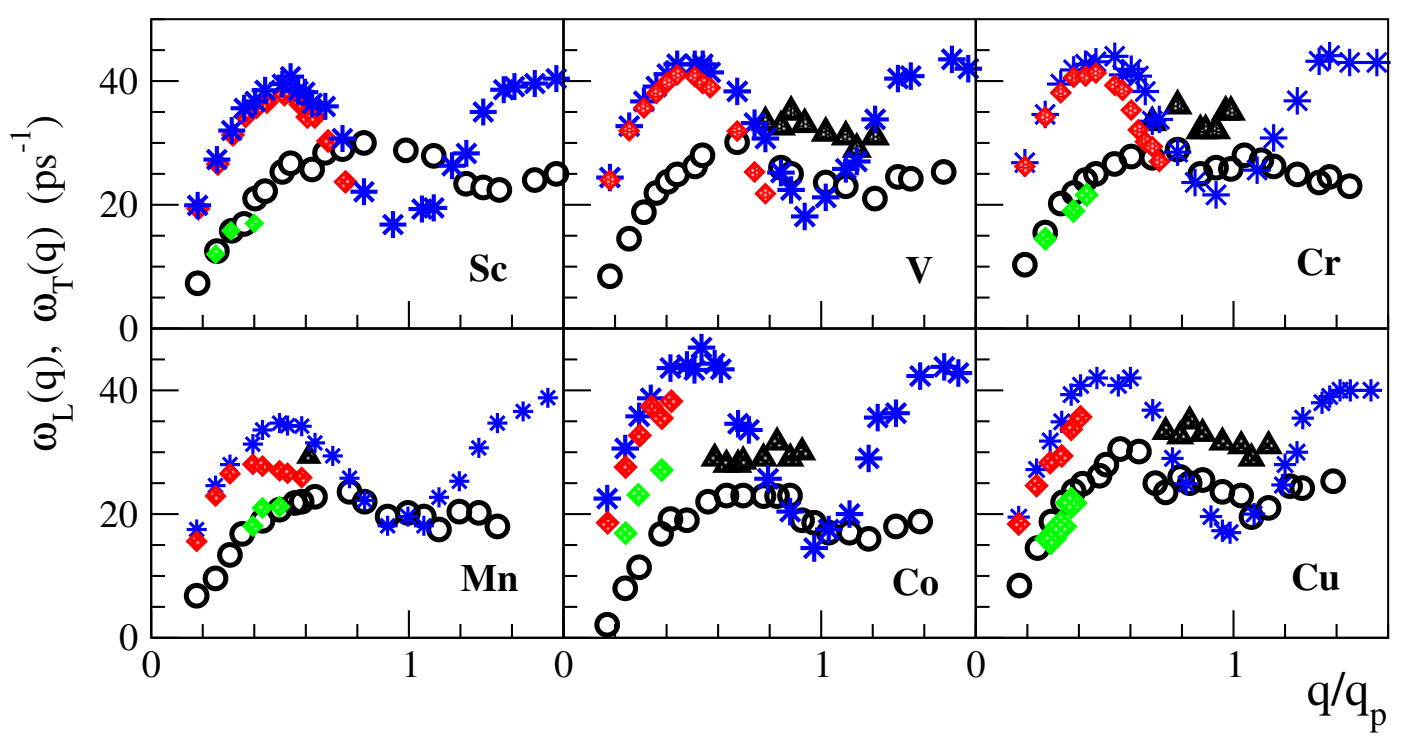

Figure 7. (Colour online) Dispersion relations for several $3 d$ liquid metals. Red diamonds and stars: longitudinal dispersion obtained from the AIMD results for the positions of the inelastic peaks in the $S(q, \omega)$ and from the maxima in the spectra of the longitudinal current, $J_{\mathrm{L}}(q, \omega)$, respectively. Open circles and triangles: transverse dispersion from the positions of the peaks in the spectra $J_{\mathrm{T}}(q, \omega)$. The green lozenges are the positions of the TA excitation modes found in the calculated dynamic structure factors, $S(q, \omega)$.

implies that the smallest attainable $q$ value, namely $q_{\min }$, is not small enough so as to permit an accurate determination of $c_{s}$; nevertheless, an approximate estimation can be obtained from value of $\omega_{m}\left(q_{\min }\right) / q_{\min }$. From the values $q_{\min }=0.43 \AA^{-1}(\mathrm{Sc}), 0.506 \AA^{-1}(\mathrm{Ti}), 0.508 \AA^{-1}(\mathrm{~V}), 0.561 \AA^{-1}(\mathrm{Cr}), 0.513 \AA^{-1}(\mathrm{Mn})$, $0.570 \AA^{-1}(\mathrm{Fe}), 0.525 \AA^{-1}(\mathrm{Co}), 0.547 \AA^{-1}(\mathrm{Ni}), 0.500 \AA^{-1}(\mathrm{Cu})$, and $0.553 \AA^{-1}(\mathrm{Zn})$, we obtained the values given in table 3 where they are compared with the available experimental/semiempirical data.

We also evaluated the longitudinal and transverse currents, $J_{\mathrm{L}}(q, t)$ and $J_{\mathrm{T}}(q, t)$, as well as their respective spectra, $J_{\mathrm{L}}(q, \omega)$ and $J_{\mathrm{T}}(q, \omega)$. The obtained $J_{\mathrm{L}}(q, \omega)$ show one peak for each $q$-value and the frequencies associated to those peaks, $\omega_{\mathrm{L}}(q)$, constitute the longitudinal dispersion relation for the associated collective modes. Figure 7 represents the obtained dispersion relations, which show the typical behaviour found in other liquid systems [35].

As for the $J_{\mathrm{T}}(q, \omega)$, it may exhibit peaks within some $q$-range, which are related to propagating shear waves. For all the metals studied in this paper, the associated $J_{\mathrm{T}}\left(q=q_{\mathrm{min}}, \omega\right)$ already showed a peak and with increasing $q$-values the associated frequency of the peak, $\omega_{\mathrm{T}}(q)$ also increased and reached a maximum value for $q \approx(2 / 3) q_{p}$; therefrom it decreased and vanished at $\approx 3.0 q_{p}$. An example of this behaviour is given in figure 8 which shows the calculated $J_{\mathrm{T}}(q, \omega)$ for 1-V and 1-Cr, as a function of $\omega$, and for a range $0<q \leqslant 4.0 \AA^{-1}$. 

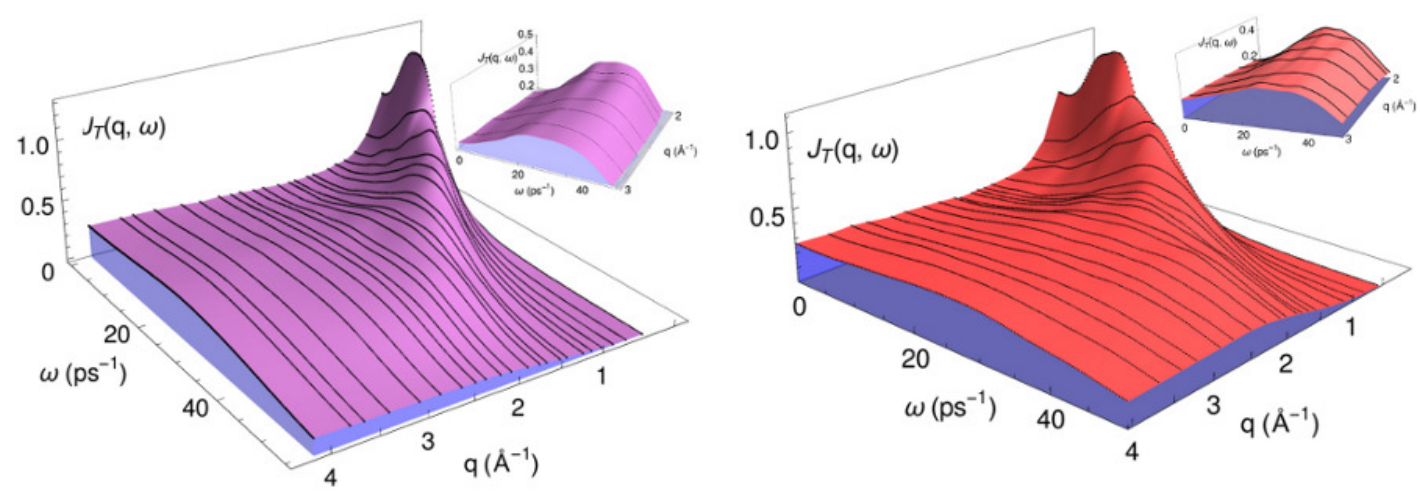

Figure 8. (Colour online) Transverse current spectra, $J_{\mathrm{T}}(q, \omega)$, of $1-\mathrm{V}$ at $T=2173 \mathrm{~K}$ and $1-\mathrm{Cr}$ at $T=2173 \mathrm{~K}$ at several $q$-values. The inset gives a detailed view of the region where the $J_{\mathrm{T}}(q, \omega)$ displays two maxima.

Moreover, for some metals (1-V, 1-Cr, 1-Co, $1-\mathrm{Cu}, 1-\mathrm{Ni}$ and $1-\mathrm{Zn}$ ) we found that the $J_{\mathrm{T}}(q, \omega)$ exhibited, within the $q$-region $0.8 \leqslant q / q_{p} \leqslant 1.2$, another peak with a higher frequency. Figure 8 shows, for $1-\mathrm{V}$ and 1-Cr, a closer view of that specific region where the two maxima are visible. For the other metals, (i.e., 1-Sc, 1-Ti, 1-Mn and 1-Fe) the results are not conclusive because, besides the lower frequency peak, we also found higher frequency shoulders instead of peaks.

The appearance of another, high frequency branch in the transvese dispersion relation, had been reported first in some liquid metals at high pressure, i.e., $\mathrm{Li}, \mathrm{Na}, \mathrm{Fe}, \mathrm{Al}$ and $\mathrm{Pb},[2,64-66$. Subsequently, it was also found in 1-Tl and 1-Pb [64, 66, 67] at ambient pressure.

From the calculated $J_{\mathrm{T}}(q, t)$, we also estimated [35] the shear viscosity coefficient $\eta$, as follows. The memory function representation of $J_{\mathrm{T}}(q, t)$, namely

$$
\tilde{J}_{\mathrm{T}}(q, z)=\frac{k_{\mathrm{B}} T}{m}\left[z+\frac{q^{2}}{\rho m} \tilde{\eta}(q, z)\right]^{-1},
$$

where the tilde denotes the Laplace transform, introduces a generalized shear viscosity coefficient $\tilde{\eta}(q, z)$. The area under the normalized $J_{\mathrm{T}}(q, t)$ gives $m \tilde{J}_{\mathrm{T}}(q, z=0) /\left(k_{\mathrm{B}} T\right)$, from which $\tilde{\eta}(q, z=0) \equiv \tilde{\eta}(q)$ can be obtained. Extrapolation to $q \rightarrow 0$ gives the standard shear viscosity coefficient $\eta$. Different extrapolation functions lead to different values of $\eta$, which are portrayed in the uncertainty assigned to the results. Table 3 shows these results along with the available experimental/semiempirical data.

Recently, it was suggested [68] that transverse-like low-energy excitations might be observed as weak shoulders located in the region between the quasielastic and the longitudinal inelastic peaks of the $S(q, \omega)$. These excitations are usually visible within a small $q$-range around $q_{p} / 2$, because for smaller/greater $q$-values they are overcome by the quasielastic/inelastic peaks. These transverse-like excitations were first detected in the IXS spectra of 1-Ga [68] at $313 \mathrm{~K}$ and subsequently in 1-Cu, 1-Sn, 1-Na, 1-Fe, 1-Ni and 1-Zn [3, 29, 30, 69. 71]. We analyzed the results for $S(q, \omega)$ and found that within the range $1.00 \AA^{-1} \leqslant q \leqslant$ $1.45 \AA^{-1}$, some weak shoulders appear in the $\omega$-region located between the quasielastic and the inelastic peaks. This is shown in figure 9; moreover, the energies associated to these shoulders are close to those corresponding to the peaks in the transverse current spectra. The real physical origin of these excitations is still a matter of debate, and some alternative interpretations, for instance heat waves, were proposed within the framework of the GCM approach [72].

\subsection{Electronic properties: density of states}

The partial and total electronic density of states, $n(E)$, were obtained from the self-consistently determined eigenvalues; they were averaged over four/six ionic configurations well separated in time $(\approx 15.0 \mathrm{ps})$, and the sampling of the Brillouin zone was performed using an $8 \times 8 \times 8$ Monkhorst-Pack set.

Figure 10 shows the obtained results for the electronic total $n(E)$ associated to the outer valence electrons, which is dominated by the $3 d$ states, except in the early transition elements, where the $d$ band 


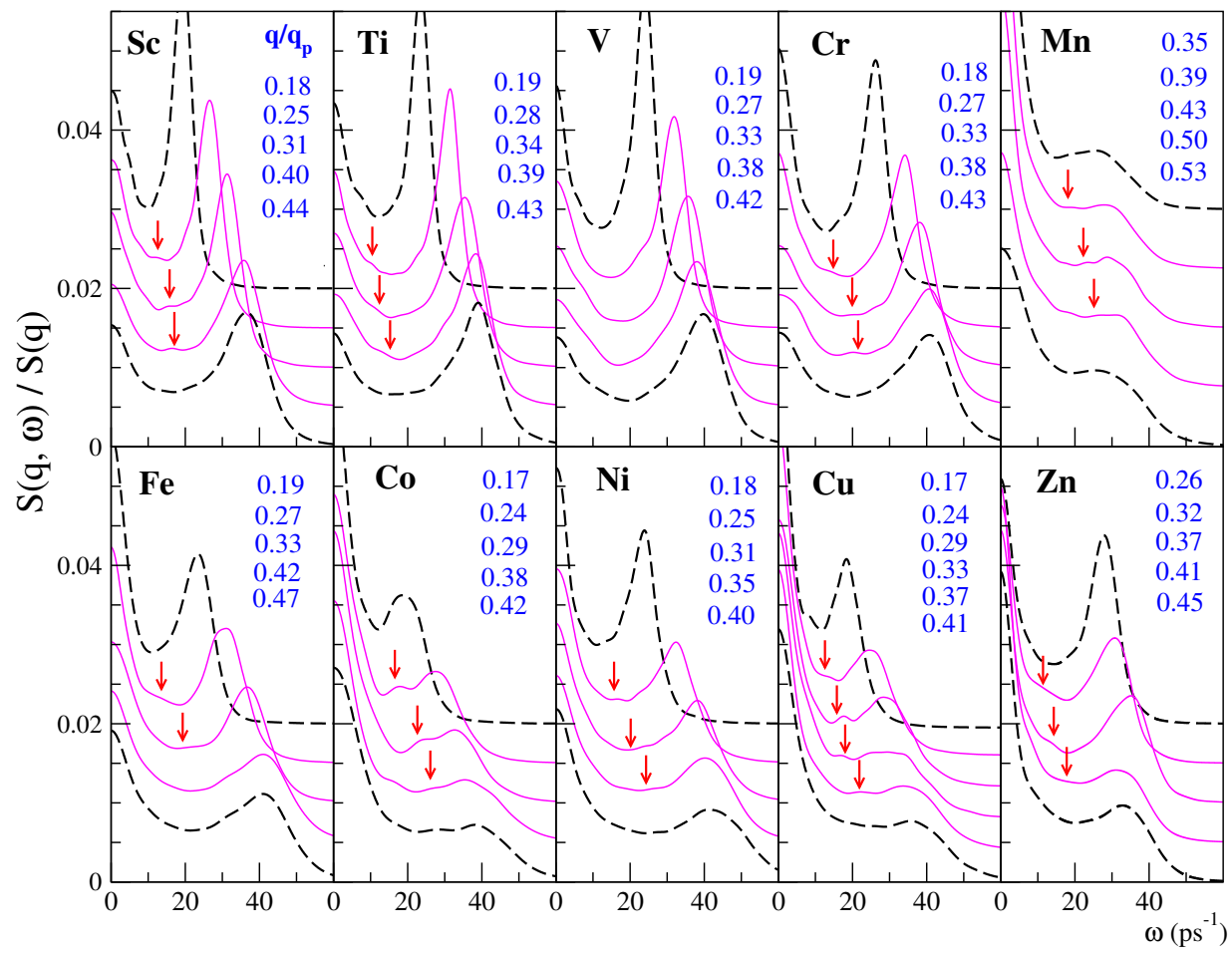

Figure 9. (Colour online) AIMD calculated dynamic structure factors $S(q, \omega) / S(q)$ of the $3 d$ liquid transition metals. They are plotted for different values of $q / q_{p}$ (top to bottom). The vertical scales are offset for clarity. The arrows point to the locations of the transverse-like excitations.

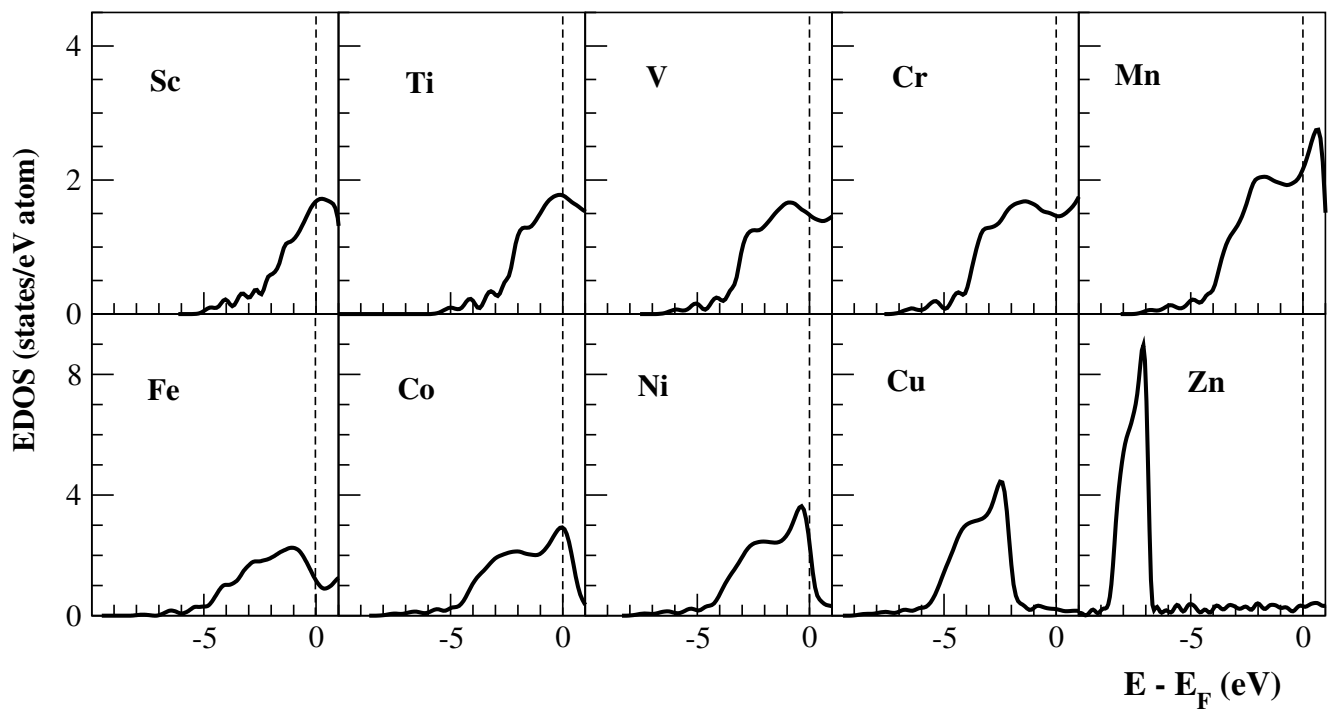

Figure 10. Total electronic density of states. Notice the different scale in the upper and lower panels.

occupation is low. Notice that for the metals $\mathrm{Sc}$ to $\mathrm{Mn}$ we are not showing the contribution from the $3 \mathrm{~s}$ and $3 p$ atomic states, which were included in the self-consistent calculation but lie way below the Fermi energy. In the figure we observe the progresive filling of the $d$ band, which is complete at $\mathrm{Cu}$, together with a decrease of its width and an increase of its height as the occupation rises. 


\section{Conclusions}

An ab initio molecular dynamics simulation method was used to calculate several static and dynamic properties of the bulk liquid $3 d$ transition metals near their respective melting points.

The results for the static structure, as characterized by the static structure factor $S(q)$, show a good agreement with the available experimental data. The only exception is 1-Sc where a clear difference is observed in the phase of the oscillations, but this may be due to inaccuracies in the old experimental data, similar to the case of the old data for 1-Ti. The $S(q)$ display, in most cases, an asymmetric shape in the second peak which qualitatively agrees with the recent ND and/or XD data. This feature is related to the appearance of icosahedral short-range order in the liquid metal. This is confirmed by a more detailed study of the liquid static structure by using the CNA method, which revealed a marked abundance of five-fold structures. On the other hand, secondary local structures also present in the liquid correlate rather well with the solid phase from where the metals melt.

The calculated dynamic structure factors, $S(q, \omega)$, show side-peaks which are indicative of collective density excitations. A detailed analysis of the $S(q, \omega)$ revealed some type of excitations which have similar features as the transverse-like excitation modes found in IXS and INS data for several liquid metals near their respective melting points.

From the calculated longitudinal and transverse current correlation functions, we evaluated the associated spectral functions and the corresponding dispersion relations. For some metals, we obtained two branches of transverse modes, with the high frequency branch appearing over a limited $q$-range which is always located in the second pseudo-Brillouin zone.

Results are also reported for several transport coefficients such as the self-diffusion, adiabatic sound velocity and shear viscosity. Although the calculated values for the adiabatic sound velocity show a reasonable agreement with the available experimental data, the lack of experimental data for the selfdiffusion and the shear viscosity provides a further relevance of these results.

\section{Acknowledgements}

This work has been supported by Junta de Castilla y Leon (project VA124G18). LEG and DJG also acknowledge the funding from the Spanish Ministry of Economy and Competitiveness (Project PGC2018-093745-B-I00), which is partially supported by the EU FEDER program.

\section{References}

1. Del Rio B.G., Rodriguez O., González L.E., González D.J., Comput. Mater. Sci., 2017, 139, 243, doi $10.1016 /$ j.commatsci.2017.07.027

2. Marques M., González L.E., González D.J., Phys. Rev. B, 2015, 92, 134203, doi:10.1103/PhysRevB.92.134203

3. Del Rio B.G., González D.J., González L.E., J. Chem. Phys., 2017, 146, 034501, doi:10.1063/1.4973803.

4. Del Rio B.G., González L.E., Phys. Rev. B, 2017, 95, 224201, doi 10.1103/PhysRevB.95.224201

5. Del Rio B.G., Pascual C., González D.J., González L.E., J. Phys.: Condens. Matter, 2020, 32, 214005, doi $10.1088 / 1361-648 X / a b 6 f 16$

6. Waseda Y., The Structure of Non-Crystalline Materials, McGraw-Hill, New York, 1980.

7. Schenk T., Holland-Moritz D., Simonet V., Bellisent R., Herlach D.M., Phys. Rev. Lett., 2002, 89, 075507, doi $10.1103 /$ PhysRevLett.89.075507

8. Inui M., Maruyama K., Kajihara Y., Nakada M., Phys. Rev. B, 2009, 80, 180201, doi 10.1103/PhysRevB.80.180201.

9. Lee G.W., Gangopadhyay A.K., Kelton K.F., Hyers R.W., Rathz T.J., Rogers J.R., Robinson D.S., Phys. Rev. Lett., 2004, 93, 037802, doi:10.1103/PhysRevLett.93.037802

10. Holland-Moritz D., Heinen O., Bellissent R., Schenk T., Mater. Sci. Eng., A, 2007, 449-451, 42, doi $10.1016 /$ j.msea.2005.12.093

11. Lou H., Wang X., Cao Q., Zhang D., Zhang J., Hu T., Mao H.-K., Jiang J.-Z., Proc. Natl. Acad. Sci. U.S.A., 2013, 110, 10068, doi $10.1073 /$ pnas.1307967110

12. Lee G.W., Gangopadhyay A.K., Hyers R.W., Rathz T.J., Rogers J.R., Robinson D.S., Goldman A.I., Kelton K.F., Phys. Rev. B, 2008, 77, 184102, doi:10.1103/PhysRevB.77.184102 
13. Blairs S., Int. Mater. Rev., 2007, 52, 321, doi:10.1179/174328007X212490

14. Casas J., Keita N.M., Steinemann S.G., Phys. Chem. Liq., 1984, 14, 155, doi 10.1080/00319108408080806

15. Assael M.J., Kakosimos K., Banish R.M., Brillo J., Egry I., Brooks R., Quested P.N., Mills K.C., Nagashima A., Sato Y., Wakeham W.A., J. Phys. Chem. Ref. Data, 2006, 35, 285, doi $10.1063 / 1.2149380$

16. Egry I., Scr. Metall. Mater., 1993, 28, 1273, doi 10.1016/0956-716X(93)90467-7

17. Said A.H., Sinn H., Alatas A., Burns C.A., Price D.L., Saboungi M.L., Schirmacher W., Phys. Rev. B, 2006, 74, 172202, doi:10.1103/PhysRevB.74.172202.

18. Agaev A.D., Kostikov V.I., Bobkovskii V.N., Izv. Akad. Nauk S.S.S.R., Ser. Met., 1980, 3, 43.

19. Iida T., Guthrie R.I.L., The Thermophysical Properties of Metallic Liquids, Oxford University Press, Oxford, 2015.

20. Nash P.M., Manghnani M.H., Secco R.A., J. Geophys. Res. Solid Earth, 1994, 99, 4285, doi 10.1029/93JB03111

21. Shimoji M., Itami T., Atomic Transport in Liquid Metals, Trans-Tech Publications, Brookfield, 1986.

22. Hirai M., ISIJ Int., 1993, 33, 251, doi 10.2355/isijinternational.33.251

23. Nachtrieb N.H., Fraga E., Wahl C., J. Phys. Chem., 1963, 67, 2353, doi:10.1021/j100805a022

24. Assael M.J., Armyra I.J., Brillo J., Stankus S.V., Wu J., Wakeham W.A., J. Phys. Chem. Ref. Data, 2012, 41, 033101, doi: $10.1063 / 1.4729873$.

25. Marcus Y., J. Chem. Thermodyn., 2017, 109, 11, doi 10.1016/j.jct.2016.07.027

26. Hosokawa S., Inui M., Matsuda K., Ishikawa D., Baron A.Q.R., Phys. Rev. B, 2008, 77, 174203, doi 10.1103/PhysRevB.77.174203.

27. Johnson M.W., McCoy B., March N.H., Page D.I., Phys. Chem. Liq., 1977, 6, 243, doi $10.1080 / 00319107708084143$.

28. Bermejo F.J., Saboungi M.L., Price D.L., Alvarez M., Roessli B., Cabrillo C., Ivanov A., Phys. Rev. Lett., 2000, 85, 106, doi: 10.1103/PhysRevLett.85.106

29. Hosokawa S., Inui M., Kajihara Y., Tsutsui S., Baron A.Q.R., J. Phys.: Condens. Matter, 2015, 27, 194104, doi $10.1088 / 0953-8984 / 27 / 19 / 194104$

30. Zanatta M., Sacchetti F., Guarini E., Orecchini A., Paciaroni A., Sani L., Petrillo C., Phys. Rev. Lett., 2015, 114, 187801, doi:10.1103/PhysRevLett.114.187801

31. Horbach J., Rozas R.E., Unruh T., Meyer A., Phys. Rev. B, 2009, 80, 212203, doi:10.1103/PhysRevB.80.212203

32. Chathoth S.M., Meyer A., Koza M.M., Juranyi F., Appl. Phys. Lett., 2004, 85, 4881, doi 10.1063/1.1825617.

33. Meyer A., Phys. Rev. B, 2010, 81, 012102, doi $10.1103 /$ PhysRevB.81.012102

34. Pasquarello A., Laasonen K., Car R., Lee C., Vanderbilt D., Phys. Rev. Lett., 1992, 69, 1982, doi $10.1103 /$ PhysRevLett.69.1982

35. Balucani U., Zoppi M., Dynamics of the Liquid State, Clarendon, Oxford, 1994

36. Kresse G., Hafner J., Phys. Rev. B, 1993, 48, 13115, doi:10.1103/PhysRevB.48.13115

37. Jakse N., Pasturel A., Condens. Matter Phys., 2015, 18, 43603, doi:10.5488/CMP.18.43603

38. Jakse N., Pasturel A., J. Chem. Phys., 2004, 120, 6124, doi $10.1063 / 1.1651054$

39. Jakse N., Wax J.F., Pasturel A., J. Chem. Phys., 2007, 126, 234508, doi:10.1063/1.2741521

40. Ganesh P., Widom M., Phys. Rev. B, 2008, 77, 014205, doi 10.1103/physrevb.77.014205

41. Del Rio B.G., Chen M., González L.E., Carter E.A., J. Chem. Phys., 2018, 149, 094504, doi 10.1063/1.5040697

42. Hohenberg P., Kohn W., Phys. Rev., 1964, 136, B864, doi 10.1103/PhysRev.136.B864

43. Kohn W., Sham L.J., Phys. Rev., 1965, 140, A1133, doi 10.1103/PhysRev.140.A1133.

44. Giannozzi P., Baroni S., Bonini N., Calandra M., Car R., Cavazzoni V., Ceresoli D., Chiarotti G.L., Cococcioni M., Dabo I., J. Phys.: Condens. Matter, 2009, 21, 395502, doi 10.1088/0953-8984/21/39/395502

45. Giannozzi P., Andreussi O., Brumme T., Bunau O., Buongiorno Nardelli M., Calandra M., Car R., Cavazzoni C., Ceresoli D., Cococcioni M., J. Phys.: Condens. Matter, 2017, 29, 465901, doi 10.1088/1361-648X/aa8f79

46. Kresse G., Hafner J., Phys. Rev. B, 1993, 47, 558(R), doi 10.1103/PhysRevB.47.558

47. Kresse G., Hafner J., Phys. Rev. B, 1994, 49, 14251, doi 10.1103/physrevb.49.14251

48. Kresse G., Furthmuller J., Comput. Mater. Sci., 1996, 6, 15, doi $10.1016 / 0927-0256(96) 00008-0$

49. Kresse G., Furthmuller J., Phys. Rev. B, 1996, 54, 11169, doi 10.1103/PhysRevB.54.11169

50. Perdew J.P., Burke K., Ernzerhof M., Phys. Rev. Lett., 1996, 77, 3865, doi:10.1103/PhysRevLett.77.3865

51. Perdew J.P., Wang Y., Phys. Rev. B, 1992, 45, 13244, doi $10.1103 /$ PhysRevB.45.13244

52. Perdew J.P., Zunger A., Phys. Rev. B, 1981, 23, 5048, doi 10.1103/PhysRevB.23.5048

53. Vanderbilt D., Phys. Rev. B, 1990, 41, 7892, doi 10.1103/PhysRevB.41.7892

54. Blöchl P.E., Phys. Rev. B, 1994, 50, 17953, doi $10.1103 /$ PhysRevB.50.17953

55. Kresse G., Joubert D., Phys. Rev. B, 1999, 59, 1758, doi 10.1103/PhysRevB.59.1758

56. Bhuiyan G.M., Bretonnet J.L., Gonzalez L.E., Silbert M., J. Phys.: Condens. Matter, 1992, 4, 7651, doi $10.1088 / 0953-8984 / 4 / 38 / 002$

57. Bhuiyan G.M., Silbert M., Stott M.J., Phys. Rev. B, 1996, 53, 636, doi $10.1103 /$ PhysRevB.53.636 
58. Hausleitner C., Kahl G., Hafner J., J. Phys.: Condens. Matter, 1991, 3, 1589, doi 10.1088/0953-8984/3/11/017

59. Todd J.R., Brown J.S., Phys. Lett. A, 1976, 59, 302, doi:10.1016/0375-9601(76)90801-X.

60. Kim T.H., Kelton K.F., J. Chem. Phys., 2007, 126, 054513, doi:10.1063/1.2431173

61. Jakse N., Pasturel A., Phys. Rev. Lett., 2003, 91, 195501, doi 10.1103/PhysRevLett.91.195501

62. Jakse N., Le Bacq O., Pasturel A., J. Non-Cryst. Solids, 2007, 353, 3684, doi 10.1016/j.jnoncrysol.2007.05.131

63. Honeycutt J.D., Andersen H.C., J. Phys. Chem., 1987, 91, 4950, doi 10.1021/j100303a014.

64. Bryk T., Jakse N., J. Chem. Phys., 2019, 151, 034506, doi:10.1063/1.5099099.

65. Bryk T., Ruocco G., Scopigno T., Seitsonen A.P., J. Chem. Phys., 2015, 143, 104502, doi 10.1063/1.4928976

66. Bryk T., Demchuk T., Jakse N., Phys. Rev. B, 2019, 99, 014201, doi 10.1103/PhysRevB.99.014201

67. Bryk T., Demchuk T., Jakse N., Wax J.F., Front. Phys., 2018, 6, 00006, doi:10.3389/fphy.2018.00006

68. Hosokawa S., Inui M., Kajihara Y., Matsuda K., Ichitsubo T., Pilgrim W.C., Sinn H., González L.E., González D.J., Tsutsui S., Baron A.Q.R., Phys. Rev. Lett., 2009, 102, 105502, doi $10.1103 /$ PhysRevLett.102.105502

69. Hosokawa S., Munejiri S., Inui M., Kajihara Y., Pilgrim W.C., Baron A.Q.R., Shimojo F., Hoshino K., AIP Conf. Proc., 2013, 1518, 695, doi 10.1063/1.4794661

70. Hosokawa S., Munejiri S., Inui M., Kajihara Y., Pilgrim W.C., Ohmasa Y., Sutsui T., Baron A.Q.R., Shimojo F., Hoshino K., J. Phys.: Condens. Matter, 2013, 25, 112101, doi $10.1088 / 0953-8984 / 25 / 11 / 112101$

71. Giordano V.M., Monaco G., Proc. Natl. Acad. Sci. U.S.A., 2010, 107, 21985, doi:10.1073/pnas.1006319107.

72. Bryk T., Wax J.F., J. Chem. Phys., 2016, 144, 194501, doi $10.1063 / 1.4948709$

\title{
Визначення з перших принципів деяких статичних $\mathbf{i}$ динамічних властивостей $3 d$ рідких перехідних металів поблизу плавлення
}

\author{
Б.Г. дель Ріо, К. Паскуаль, О. Родрігес, Л.Є. Гонзалес, Д.Дж. Гонзалес \\ Відділ теоретичної фізики, університет Вальядоліда, 47011 Вальядолід, Іспанія
}

\begin{abstract}
Ми повідомляємо an initio дослідження симуляціями молекулярної динаміки деяких статичних і динамічних властивостей $3 d$ рідких перехідних металів. Обчислені статичні структурні фактори показують якісне узгодження з наявними експериментальними даними, а їх другий пік має асиметричну форму, яка передбачає, що існує значний локальний ікосаедричний порядок. Динамічна структура виявляє розповсюдження флуктуацій густини, дисперсійне співвідношення яких було обчислено; крім того їх довгохвильова границя $\epsilon$ сумісною з відповідною їм експериментальною швидкістю звуку. Представлено результати для спектральних функцій і для відповідних дисперсійних співвідношень. Ми також аналізуємо можливу появу поперечно-подібних низькоенергетичних збуджень в обчислених динамічних структурних факторах. Обчислено декілька коефіцієнтів переносу і порівняно з наявними експериментальними даними.
\end{abstract}

Ключові слова: рідкі метали, першопринципні розрахунки 